\title{
A Pareto Optimal Auction Mechanism for Carbon Emission Rights
}

\author{
Mingxi Wang, ${ }^{1}$ Mingrong Wang, ${ }^{2}$ Chuangyin Dang, ${ }^{3}$ and Shouyang Wang ${ }^{4}$ \\ ${ }^{1}$ School of International Trade and Economics, University of International Business and Economics, Beijing 100029, China \\ ${ }^{2}$ School of Economics, Capital University of Economics and Business, Beijing 100070, China \\ ${ }^{3}$ Department of Manufacturing Engineering \& Engineering Management, City University of Hong Kong, Kowloon, Hong Kong \\ ${ }^{4}$ Academy of Mathematics and Systems Science, Chinese Academy of Sciences, Beijing 100190, China
}

Correspondence should be addressed to Mingxi Wang; mxwang@amss.ac.cn

Received 23 December 2013; Accepted 24 February 2014; Published 30 March 2014

Academic Editor: Chuangxia Huang

Copyright (C) 2014 Mingxi Wang et al. This is an open access article distributed under the Creative Commons Attribution License, which permits unrestricted use, distribution, and reproduction in any medium, provided the original work is properly cited.

\begin{abstract}
The carbon emission rights do not fit well into the framework of existing multi-item auction mechanisms because of their own unique features. This paper proposes a new auction mechanism which converges to a unique Pareto optimal equilibrium in a finite number of periods. In the proposed auction mechanism, the assignment outcome is Pareto efficient and the carbon emission rights' resources are efficiently used. For commercial application and theoretical completeness, both discrete and continuous marketsrepresented by discrete and continuous bid prices, respectively-are examined, and the results show the existence of a Pareto optimal equilibrium under the constraint of individual rationality. With no ties, the Pareto optimal equilibrium can be further proven to be unique.
\end{abstract}

\section{Introduction}

An emission-right market is an economic instrument to correct the market failure caused by negative environmental externality. The study of the emission-right market has long been a major issue in economic research (in the 1960s, Coase [1], Crocker [2], and Dales [3] proposed the seminal idea of an emission-right market; in 1988, Baumol and Oates [4] developed the theoretical framework for the emissionright market) and blossomed since the conference of the Parties of Kyoto in 1997 (see, e.g., Linares and Romero [5], Georgopoulou et al. [6], Nordhaus [7], Jotzo and Pezzey [8], Tol [9], Zhang [10], and Burniaux and Oliveira Martins [11]). To meet the goal of carbon emission reduction in the Kyoto Protocol, European Union in 2005 established the auctionand-trade scheme which makes the carbon emission right a tradable commodity. (The auction-and-trade scheme was initially known as the emission-and-trade scheme. With time, the percentage of carbon emission right traded is fast increasing by auction. Capoor and Ambrosi [12] estimated that $70-80 \%$ of carbon emission allowances would be auctioned by 2020 . Therefore, the emission-and-trade scheme is often called to be the auction-and-trade scheme.)

The auction-and-trade scheme provides one way of addressing the question of price formation of carbon emission rights; however it fails to answer such an important and practical question as whether or not the assignment outcome is efficient. Krishna [13] demonstrated in a single item setting that the assignment outcomes closely depend on the auction mechanism design and the resale after the auction sometimes fails to attain efficient outcomes. In multiple items situation, Milgrom [14] mentioned that an inefficient initial assignment cannot, in general, be quickly corrected by trading in items after the auction is complete. Therefore, it is both interesting and challenging to design an auction mechanism to attain an efficient assignment in the auction-and-trade scheme.

The existing auction mechanisms for multiple items (such as Ausubel [15], Mishra and Garg [16], Mishra and Veeramani [17], Avenali [18], Choi et al. [19], Sun and Yang [20], Goel et al. [21], Shih et al. [22], and Liu [23]) are inappropriate for carbon emission rights due to their unique features. On one hand, the carbon emission right bears a strong resemblance to 
the ordinary commodity. Given the production technology, the producer, who uses more carbon emission rights as an input factor in the production, can emit more $\mathrm{CO}_{2}$ and further produces more outputs. On the other hand, the carbon emission right is significantly different from the ordinary commodity. In the auction-and-trade scheme, the carbon emission right is transferred from the auctioneer (or government) to bidders (or companies). The bidder, winning the right, pays for it at the auction price to the auctioneer. However, the auctioneer obtains the environmental profit (or more clean air) from carbon emission reduction relative to the business-as-usual case, other than the auction price. Besides, the carbon emission right has a limited duration of validity and its value will expire after commitment period. Meanwhile, the trade of carbon emission right influences the industrial structure by bidders' decision. (Carbon emissions are caused by the fuel of carbon-based energy. Thus, if the price of carbon emission right is high, emitting entities may change their production process in two aspects. On one hand, to reduce cost and obtain certain profit, they may change their input combinations by replacing carbon-based energy with clean energy. On the other hand, to remain in business, entities may change their outputs by creating some new "green goods." Subsequently, the associated upriver and downriver industries must do corresponding adjustments. Then, the industrial structure will change.) Thus, the auction mechanism design for carbon emission rights will be different from that for ordinary items.

Such unique features make the auction mechanism design problem as to carbon emission rights allowance become more difficult. Generally speaking, a series of papers have been published on carbon emission rights auctions in the management science and operation research literature, but from the perspective of arguing EU ETS (Emissions Trading Scheme). In particular, Benz and Truck [24] describes the issue concerning how carbon emission rights become a class of tradable assets, Benz et al. [25] and Cramton and Kerr [26] analyze alternative initial allowance methods of carbon emission rights (auctioning or grandfathering), and Capoor and Ambrosi [12], Grubb [27], Clò [28], and Kruger [29] comment on the efficiency and effectiveness of carbon emission reduction in the ETS. However, these papers focus on the management performance of the ETS and its implementability, leaving open associated auction mechanism design problem. Burtraw et al. [30] and Holt et al. [31] are among the few papers that investigate the auction mechanism design problem of carbon emission rights. Yet, there is no paper that examines the problem from the viewpoint of existence and uniqueness of equilibrium.

Enlightening by the tatonnement process, we develop a Pareto optimal auction mechanism for carbon emission rights. Several issues arise. The first one is how to design auction rules to make the final assignment converge to a Pareto optimal equilibrium. It is interesting that in proposed mechanism, the market is cleared by a simultaneous lowering of the "expectations" of bidders and raising of the auctioneer's "expectation." At equilibrium, there are no coalitions that can improve the auctioneer's and bidders' utility simultaneously. Therefore, we call the mechanism the Pareto optimal auction.
Besides, the proposed mechanism, consisting of four steps, is quite easy and simple to use by players and more practical to future commercial applications.

The second issue concerns the existence of equilibrium in the Pareto optimal auction. For practical use, we first examine the discrete market where the bid price changes discretely. Under the constraint of individual rationality, the discrete market converges to a discrete Pareto optimal equilibrium in a finite number of periods. For the sake of comparison with the discrete market, we then discuss the existence of Pareto optimal equilibrium in the continuous market where the bid price changes continuously. Surprisingly, the existence of equilibrium in the discrete market provides a proof of equilibrium existence in the continuous market.

The third issue is the uniqueness of equilibrium in the Pareto optimal auction. In the two-side market, there are often multiple equilibria in Gul and Stacchetti [32], which makes equilibrium depend on the choice of price adjustment process. In case of a discrete market with no ties, we prove that the Pareto optimal auction converges to a unique strong Pareto optimal equilibrium.

The paper proceeds as follows. Section 2 describes the model. Section 3 designs the Pareto optimal auction mechanism and examines the existence and uniqueness of its equilibrium. Section 4 concludes this paper.

\section{The Model}

Suppose there are $m$ indivisible carbon emission rights and $n$ bidders indexed by $i=1,2, \ldots, m$ and $j=1,2, \ldots, n$, respectively. The carbon emission rights are assigned by the auctioneer to the bidders who want to purchase a given quantity of carbon emission rights. The auctioneer's utility and bidders' profit are realized across carbon emission rights transfer. If bidder $j$ wins right $i$ at the bid price of $b_{i j}$, the utility of the auctioneer consists of the bid price and the environmental benefit:

$$
u_{i j}=a_{i j}+b_{i j},
$$

where $u_{i j}$ is the utility function and $a_{i j}$ the cost to protect the environment; the $j$ th bidder's profit is

$$
v_{i j}=p_{i j}-b_{i j},
$$

where $v_{i j}$ is the $j$ th bidder's profit and $p_{i j}$ the productivity of right $i$ to bidder $j$. (In reality, the environmental benefit is endogenously determined by the whole economy. Therefore, the product functions of bidders (e.g., $\left.f_{j}(i)\right)$ should be given. In this paper, we assume that the environmental benefit and the productivity of emission right are independent. However, doing so is without loss of generality, because all lemmas and theorems still hold with $f_{j}(i)$ replacing of $p_{i j}$. Here, to simplify notations, we choose $p_{i j}$.) It is further assumed that $a_{i j}+p_{i j} \geq 0$ for all $(i, j)$. This assumption guarantees the utility gains for either the bidder or the auctioneer; otherwise the auction would be aborted. The utility or profit of doing nothing is normalized as zero. Let $b_{i j}(t)$ be the bid price that the $j$ th bidder is permitted to make to the $i$ th right at time $t$. 
In the discrete case, we assume that $a_{i j}$, $p_{i j}$, and $b_{i j}(t)$ are integers, which can be satisfied for the sufficiently small unit of measurement. The continuous case will be theoretically analyzed later by making use of the results in the discrete case. It is natural to assume that bidders buy many carbon emission rights and that each right is assigned only to one bidder. For notational simplicity, we assume that each bidder wants exactly to buy one right and each right is assigned exactly to one bidder. However, the arguments here can be extended to the general case almost without modification.

In general, $m<n$. For the convenience of the following passage, the dummy carbon emission rights are introduced to make $m=n$. Since the dummy rights make no contribution to productivity, their bid prices are null. It immediately follows that the assignment rule $\phi:\{1,2, \ldots, n\} \rightarrow\{1,2, \ldots, n\}$ is a one-to-one function, where $\phi(i)$ is the bidder to whom the $i$ th right is assigned. Surely, assignment rule $\phi$ is reversible: $\phi^{-1}(\cdot)=\psi(\cdot)$, where $\psi(j)$ is the right assigned to the $j$ th bidder. The auction outcome consists of assignment rule $\phi$ and bid prices $\left(b_{1 \phi(1)}, b_{2 \phi(2)}, \ldots, b_{n \phi(n)}\right)$.

In an exchange economy, the equilibrium under the perfect information requires that no coalitions are able to obtain mutual benefit, compared to current matched combination. Correspondingly, the assignment coincides with the Pareto optimal allocation as in Mas-Colell et al. [33, page 522]. To preserve the property of Pareto efficiency, we refer to the present notion as the Pareto optimal allocation in the auction studied below. Actually, it is useful to distinguish between the allocation in discrete market and that in the continuous market. These conditions motivate the following definitions.

Definition 1. An allocation $\left(\phi ; b_{1 \phi(1)}, b_{2 \phi(2)}, \ldots, b_{n \phi(n)}\right)$ is individually rational, if the assignment of carbon emission rights to bidders together with the bid price vector are such that

$$
\begin{gathered}
a_{i \phi(i)}+b_{i \phi(i)} \geq 0 \quad \forall i, \\
p_{\psi(j) j}-b_{\psi(j) j} \geq 0 \quad \forall j .
\end{gathered}
$$

Definition 1 is a participating constraint. It makes each bidder not worse than doing nothing and the auctioneer willing to sell off each right at given its bid price.

Definition 2. An individually rational allocation $\left(\phi ; b_{1 \phi(1)}\right.$, $\left.b_{2 \phi(2)}, \ldots, b_{n \phi(n)}\right)$ is (discrete) strong Pareto optimal, if there is no right-bidder coalition $(i, j)$ and (integer) bid price $b$ such that

$$
\begin{gathered}
a_{i j}+b \geq a_{i \phi(i)}+b_{i \phi(i)}, \\
p_{i j}-b \geq p_{\psi(j) j}-b_{\psi(j) j},
\end{gathered}
$$

with strict inequality holding for at least one of (4). An individually rational allocation $\left(\phi ; b_{1 \phi(1)}, b_{2 \phi(2)}, \ldots, b_{n \phi(n)}\right)$ is (discrete) Pareto optimal, if there is no right-bidder coalition $(i, j)$ and (integer) bid price $b$ that satisfy both (4) with strict inequality.

Definition 3. A carbon emission right $i$ is said to $b e b^{j}$-feasible for bidder $j$, if there is some discrete Pareto optimal allocation that assigns right $i$ to bidder $j$ at bid price $b^{j}$.
Coalition in Definitions 2 and 3 is said to be capable of improving upon the given allocation. It is clear from the above definitions that a strong Pareto optimal allocation is a Pareto optimal allocation. Further, it is easy to see that a (strong) Pareto optimal allocation of a continuous market at which bid prices satisfy the appropriate integer restrictions is also a (strong) Pareto optimal allocation of the corresponding discrete market. Thus, in a Pareto optimal allocation, the total utility of both the auctioneer and the bidders from all rights is maximized.

\section{The Pareto Optimal Auction Mechanism}

In this section, we first design the auction rules as simple as possible and call it the Pareto optimal auction mechanism because the auction converges to a Pareto optimal equilibrium (this will be checked in Theorems 6 and 7). For both commercial application and theoretical completeness, equilibrium existence under the proposed auction is then examined when bid prices change discretely and continuously, respectively. Finally, equilibrium uniqueness and assignment efficiency are analyzed with no-tie constraint.

Consider the following auction, defined for a discrete market on the matrix of integer bid prices $\left[b_{i j}(t)\right]$, where time $t$ is measured discretely.

Step 1. The auctioneer announces an initial price matrix $\left[b_{i j}(0)=-a_{i j}\right]$ for all $(i, j)$. Let $t:=0$ and go to Step 2 .

Step 2. Given the matrix of permitted prices $\left[b_{i j}(t)\right]$, each bidder makes bids to her favorite carbon emission rights. For example, bidder $j$ makes a bid to right $i$, where $i$ is a solution of the problem

$$
\max _{h}\left\{p_{h j}-b_{h j}(t)\right\} .
$$

The right which receives more bids rejects all but the auctioneer's favorite one, taking into account environmental benefit and bid prices. However, bidders and the auctioneer may break ties at any time they like. For the right which receives only one bid, no rejection happens. As the auction continues, those bids not rejected in previous periods remain constant. Otherwise, the auction goes to Step 3 .

Step 3. If the auctioneer rejects a bid from bidder $j$ in period $t-1, b_{i j}(t)=b_{i j}(t-1)+1$. Rejected bidders continue to make bids to their favorite rights based on (5), taking into account their current permitted prices.

Lemma 4. Each carbon emission right receives at least one bid during the whole period of the auction.

Proof. For any carbon emission right $i$, since $p_{i j}-b_{i j}(0)=$ $p_{i j}+a_{i j} \geq 0$ for all $j$, bidders are willing to make bids to all carbon emission rights at the beginning of the auction. Suppose that carbon emission right $i$ receives one bid $b_{i j}(0)$ from bidder $j$ at $t=0$. There are only two situations for the auctioneer. One is that the bid $b_{i j}(0)$ is not rejected until the auction ends. The other is that in some period $t$, the bid $b_{i j}(0)$ 
is rejected by the auctioneer. The only reason is that at time $t$ another bid from some bidder $k$ is made at $b_{i k}$ such that

$$
a_{i j}+b_{i j}(0) \leq a_{i k}+b_{i k}(t)
$$

where by Step $3 i$ is a solution of (5) at $t$ for bidder $k$. Thus, right $i$ has the bid from bidder $k$ instead of the one from bidder $j$. In a word, right $i$ receives at least one bid during the whole period of the auction.

Step 4. Repeating Steps 2 and 3, the auction ends until the period when no rejections are issued. The auctioneer accepts the bids from the bidders who have not been rejected.

Lemma 5. The auction ends after a finite number of periods and each (real or dummy) carbon emission right gets exactly one bid.

Proof. By Lemma 4, each right gets at least one bid at any time $t \geq 0$. If at time $t$ right $i$ receives more than one bids, $b_{i k_{1}}, b_{i k_{2}}, \ldots, b_{i k_{s}}$, it must be true that for the auctioneer,

$$
a_{i k_{1}}+b_{i k_{1}}(t)=a_{i k_{2}}+b_{i k_{2}}(t)=\cdots=a_{i k_{s}}+b_{i k_{s}}(t) .
$$

Otherwise, the auctioneer will choose her favorite one, taking environmental benefit and bid prices into account. By Step 2, without loss of generality, we suppose that at time $t^{\prime} \geq t$, the auctioneer breaks the tie by rejecting all but bidder $k_{1}$. Then, the permitted bid prices at time $t^{\prime}+1$ are $b_{i k_{2}}\left(t^{\prime}+1\right)=b_{i k_{2}}(t)+$ $1, \ldots, b_{i k_{s}}\left(t^{\prime}+1\right)=b_{i k_{s}}(t)+1$. Repeating the above argument and noting that $p_{i j}$ is finite for all $j$, bidding competition must fall, in finite time, to the point where right $i$ gets exactly one bid and no rejections are issued.

Because of the arbitrariness of right $i$ and Step 2, each carbon emission right gets exactly one bid after a finite number of periods, no rejections are issued and the auction then ends.

Based on Lemmas 4 and 5, we now establish the main theorems in this paper.

Theorem 6. The Pareto optimal auction with discrete bid prices converges to a discrete Pareto optimal equilibrium in a finite number of periods.

Proof. First, we prove that the proposed auction converges to an individually rational allocation in the discrete market. Let $t^{*}$ be the time at which the auction ends. Since $b_{i j}(0)=-a_{i j}$ for all $(i, j)$ and $b_{i j}(t)$ never falls by Step 2 and Step 3 , it is immediate that

$$
a_{i j}+b_{i j}\left(t^{*}\right) \geq 0 \quad \forall(i, j)
$$

Thus, by Definition 1 we only need to prove that

$$
p_{i j}-b_{i j}\left(t^{*}\right) \geq 0
$$

if bidder $j$ wins carbon emission right $i$ when the auction ends. Suppose that $p_{i j}-b_{i j}\left(t^{*}\right)<0$. Then, winning right $i$ makes bidder $j$ worse than doing nothing. By (5) of Step 2, bidder $j$ could not have made a bid at $b_{i j}\left(t^{*}\right)$ to right i. Considering dummy carbon emission rights, Lemma 5 implies that every right gets exactly one bid at the auction end, and it follows that right $i$ is not one that bidder $j$ wins at $t^{*}$. Hence, if bidder $j$ wins right $i$ at $t^{*}$, we must have $p_{i j}-b_{i j}\left(t^{*}\right) \geq 0$.

Second, we prove by way of contradiction that the proposed auction converges to a discrete Pareto optimal allocation in the discrete market. By Lemma 5, in a finite number of periods the auction converges to an individually rational equilibrium denoted by $\left(\phi ; b_{1 \phi(1)}, b_{2 \phi(2)}, \ldots, b_{n \phi(n)}\right)$. Suppose that $\left(\phi ; b_{1 \phi(1)}, b_{2 \phi(2)}, \ldots, b_{n \phi(n)}\right)$ is not a discrete Pareto optimal allocation. By Definition 2, there must exist a right-bidder coalition $(i, j)$ and an integer bid price $b$ such that

$$
\begin{gathered}
a_{i j}+b>a_{i \phi(i)}+b_{i \phi(i)}, \\
p_{i j}-b>p_{\psi(j) j}-b_{\psi(j) j} .
\end{gathered}
$$

For any integer $b$ satisfying (11), bidder $j$ must make a bid to right $i(i \neq \psi(j))$ at $b$ by (5). Because of $i \neq \psi(j)$, the bid by Step 3 must have been rejected by the auctioneer. Since the auctioneer never rejects those bids that are at least as good as current accepted bids, right $i$ 's equilibrium bid from bidder $\phi(i)$ at $b_{i \phi(i)}$ must satisfy

$$
a_{i \phi(i)}+b_{i \phi(i)} \geq a_{i j}+b
$$

Inequality (12) contradicts (10), and thus Theorem 6 holds.

In practice, for the sufficiently small money unit, say penny, Theorem 6 ensures that the allocation is Pareto efficient in the proposed auction. Our discussion has been confined so far to discrete markets. Since small changes in other rights' bids can induce large changes in the adjustments a right's permitted bid price must undergone, it would be of interest to know, for the sake of comparison with the discrete market, what the continuous market implies about the existence of the Pareto optimal allocation. Perhaps somewhat surprisingly, the existence of Pareto optimal allocation in the discrete market allows a proof of the existence of a strong Pareto optimal allocation in the continuous market, as we now argue.

Theorem 7. The Pareto optimal auction with continuous bid prices has a strong Pareto optimal equilibrium.

Proof. By way of contradiction, suppose that the proposed auction with continuous bid prices has no strong Pareto optimal equilibria. Considering its individually rational allocation $\left(\phi ; b_{1 \phi(1)}, b_{2 \phi(2)}, \ldots, b_{n \phi(n)}\right)$, let

$$
\begin{aligned}
g & {\left[(i, j) ; \phi ; b_{1 \phi(1)}, b_{2 \phi(2)}, \ldots, b_{n \phi(n)}\right] } \\
& =a_{i j}+p_{i j}-\left(a_{i \phi(i)}+b_{i \phi(i)}\right)-\left(p_{\psi(j) j}-b_{\psi(j) j}\right) .
\end{aligned}
$$


In other words, $g(\cdot)$ is the potential gain realizable by the right-bidder coalition $(i, j)$ and price $p_{i j}$ improving upon allocation $\left(\phi ; b_{1 \phi(1)}, b_{2 \phi(2)}, \ldots, b_{n \phi(n)}\right)$. Define

$$
\begin{aligned}
G & \left(\phi ; b_{1 \phi(1)}, b_{2 \phi(2)}, \ldots, b_{n \phi(n)}\right) \\
\quad & \max _{(i, j)} g\left[(i, j) ; \phi ; b_{1 \phi(1)}, b_{2 \phi(2)}, \ldots, b_{n \phi(n)}\right] .
\end{aligned}
$$

By the assumption and Definition $2, G\left(\phi ; b_{1 \phi(1)}, b_{2 \phi(2)}, \ldots\right.$, $\left.b_{n \phi(n)}\right)>0$; otherwise no right-bidder coalitions could improve upon that allocation.

We are going to show that $G\left(\phi ; b_{1 \phi(1)}, b_{2 \phi(2)}, \ldots, b_{n \phi(n)}\right)$ has a positive lower bound for all individually rational assignment $\phi$. Let

$$
l(\phi)=\min _{\left(b_{1 \phi(1)}, b_{2 \phi(2)}, \ldots, b_{n \phi(n)}\right)} G\left(\phi ; b_{1 \phi(1)}, b_{2 \phi(2)}, \ldots, b_{n \phi(n)}\right) .
$$

Since $a_{i j}+p_{i j} \geq 0$ and $-a_{i j} \leq b_{i j}(t) \leq p_{i j}$ for all $(i, j)$, the feasible region of (15) is a nonempty, bounded, and closed set. For given $\phi, G(\cdot)$ is continuous in $\left(b_{1 \phi(1)}, b_{2 \phi(2)}, \ldots, b_{n \phi(n)}\right)$ by the fact that the maximum of continuous functions is continuous. Therefore, $l(\phi)>0$ for given $\phi$. Define

$$
L=\min _{\phi \in \Phi} l(\phi),
$$

where $\Phi$ is the set of all one-to-one functions $\phi:\{1,2, \ldots$, $n\} \rightarrow\{1,2, \ldots, n\}$. Since $\Phi$ is a finite set, it immediately follows that $L$ is strictly positive and that $G\left(\phi ; b_{1 \phi(1)}\right.$, $\left.b_{2 \phi(2)}, \ldots, b_{n \phi(n)}\right) \geq L>0$.

Thus, for any individually rational allocation in the proposed auction with continuous bid prices, there is at least one bidder-right coalition improving upon the given allocation and potential gain is strictly positive. Note that an individually rational allocation of a continuous market at which bid prices satisfy the appropriate integer restrictions is also an individually rational allocation of the corresponding discrete market. The argument above implies that any individually rational allocation in the corresponding discrete market can also be improved upon by some bidder-right coalition and further that this discrete market has no Pareto optimal allocations, which contradicts Theorem 6 . Therefore, the Pareto optimal auction with continuous bid prices has a strong Pareto optimal equilibrium.

The main interest of Theorems 6 and 7 is that they lay the theoretical foundation for the existence of Pareto optimal allocations. From Step 1 to Step 4, the Pareto optimal auction can be viewed as an algorithm for computing optimal assignments. In particular, for the appropriate choice of the unit of measurement (calculable in advance if, e.g., $a_{i j}$ and $p_{i j}$ are known to be integers), our auction converges in finite time to a Pareto optimal allocation with assignment rule $\phi$. This is associated with the strong Pareto optimal allocation in the continuous market.

The effectiveness of the auction can be improved if Step 2 is modified as follows: at each time, every bidder can only choose one right from her favorite rights and the auctioneer can only choose one bid from more bids to right $i$. In other words, there are no ties in each period of the auction. Thus, given bid prices matrix $\left[b_{i j}(t)\right], u_{i j} \neq u_{i k}$ if $j \neq k$ and $v_{i j} \neq v_{h j}$ if $i \neq h$.

Lemma 8. In the Pareto optimal auction with no ties, the (discrete) strong Pareto optimal allocation coincides with the (discrete) Pareto optimal allocation.

Proof. The asymmetry between bidders and the auctioneer arises only because bidders make bids in the auction. Where bidders and rights interchanged everywhere in the auction, all results with bidders and the auctioneer interchanged would also be true. Thus, if either of (4) holds, the other must also hold. With no ties, the (discrete) strong Pareto optimal allocation makes no difference from the (discrete) Pareto optimal allocation in the Pareto optimal auction.

We now turn to the question whether the result in Theorem 6 remains valid when there are no ties. For the discrete market, the answer is yes, as shown by the following theorem.

Theorem 9. (i) In a discrete market with no ties, the proposed auction converges to a discrete strong Pareto optimal allocation.

(ii) That allocation for every bidder is at least as good as any other discrete strong Pareto optimal allocation of the discrete market.

(iii) That allocation for the auctioneer is at least as good as any other discrete strong Pareto optimal allocation of the discrete market.

Proof. (i) If no ties occur, it suffices to consider the discrete Pareto optimal allocation by Lemma 8 . Theorem 6 implies that (i) holds.

(ii) Suppose that the auctioneer rejects a bid to right $i$ from bidder $j$ at $b^{j}$ in favor of a bid from bidder $k$ at $b^{k}$. We will prove that right $i$ is not $b^{j}$-feasible for bidder $j$.

As ties have been ruled out, Step 2 implies that

$$
a_{i k}+b^{k}>a_{i j}+b^{j} .
$$

Further, by (5), right $i$ at $b^{k}$ is better for bidder $k$ than any other right at current permitted bid prices. Thus, if $h \neq i$,

$$
p_{i k}-b^{k}>p_{h k}-b^{\prime},
$$

where $b^{\prime}$ is current bid price that bidder $k$ is permitted to make to right $h$.

Consider an allocation that assigns right $i$ to bidder $j$ at $b^{j}$ and all other rights to bidders at the feasible bid prices. By (17) and (18), the auctioneer and bidder $k$ both prefer each other at integer bid price $b^{k}$ to such an allocation. Hence, it is not a discrete Pareto optimal allocation, and further right $i$ is not $b^{j}$-feasible for bidder $j$.

The above argument shows that in the auction, no bidders are ever rejected by the auctioneer at the $b$-feasible bid price for bidder $j$. By Definition 3, the allocation in (i) is $b$-feasible for every bidder. Thus, the auctioneer accepts the allocation 
outcome in (i). From the fact that bidders make bids to their favorite rights first, it therefore follows that bidders unanimously prefer the discrete Pareto optimal allocation in the proposed auction to any other discrete Pareto optimal allocation in the discrete market.

(iii) The proof of Lemma 8 demonstrates that all results will also be true with bidders and the auctioneer interchanged. From (ii), we immediately get (iii).

Theorem 9 implies that the Pareto optimal auction is not only efficient for bidders (because it maximizes bidders' profit), but also optimal for the auctioneer (because the final assignment outcome maximizes the auctioneer's utility). In this sense, our auction mechanism solves the open question of the conflict between efficiency and revenue in multi-item auctions left by Milgrom [14].

Theorem 10. In a discrete market with no ties, the proposed auction converges to unique discrete strong Pareto efficient allocation.

Proof. Suppose there are two discrete strong Pareto optimal allocations in a discrete market with no ties: $\left(\phi_{1} ; b_{1 \phi_{1}(1)}\right.$, $\left.b_{2 \phi_{1}(2)}, \ldots, b_{n \phi_{1}(n)}\right)$ and $\left(\phi_{2} ; b_{1 \phi_{2}(1)}, b_{2 \phi_{2}(2)}, \ldots, b_{n \phi_{2}(n)}\right)$; we are going to prove these two allocations are the same. For bidders, Theorem 9 (ii) implies that $v_{\psi_{1}(j) j} \geq v_{\psi_{2}(j) j}$ and $v_{\psi_{1}(j) j} \leq$ $v_{\psi_{2}(j) j}$, where $\psi_{1}=\phi_{1}^{-1}$ and $\psi_{2}=\phi_{2}^{-1}$, and thus $v_{\psi_{1}(j) j}=$ $v_{\psi_{2}(j) j}$. Further, the no ties condition implies $\psi_{1}=\psi_{2}$, and thus $\phi_{1}=\phi_{2}$. Similarly, for the auctioneer, by Theorem 9 (iii) we have $a_{i \phi_{1}(i)}+b_{i \phi_{1}(i)}=u_{i \phi_{1}(i)}=u_{i \phi_{2}(i)}=a_{i \phi_{2}(i)}+b_{i \phi_{2}(i)}$. Noted that $a_{i \phi_{1}(i)}=a_{i \phi_{1}(i)}$ for all $i$ by $\phi_{1}=\phi_{2}$, it follows that $b_{i \phi_{1}(i)}=$ $b_{i \phi_{2}(i)}$ and that $\left(\phi_{1} ; b_{1 \phi_{1}(1)}, b_{2 \phi_{1}(2)}, \ldots, b_{n \phi_{1}(n)}\right)$ and $\left(\phi_{2} ; b_{1 \phi_{2}(1)}\right.$, $\left.b_{2 \phi_{2}(2)}, \ldots, b_{n \phi_{2}(n)}\right)$ are the same. Thus, the proposed auction converges to unique discrete strong Pareto efficient allocation in a discrete market with no ties.

It is often of great interest to ask whether an economic system is producing an "optimal" economic outcome. In our proposed auction, Theorem 10 shows that the answer is yes in the sense that at equilibrium, there is no such an alternative way to redistribute carbon emission rights that makes some agent (auctioneer or bidder) better off without making some other agent worse off. Therefore, the allocation that is Pareto optimal (or Pareto efficient) uses carbon emission rights' resources efficiently.

\section{Conclusion}

Relatively to ordinary tradable goods and services, carbon emission rights have own unique features, such as the positive externality, a limited duration of validity, the effects of industrial structure, and the necessary input factor for the product process using carbon-based energy as fuel. Such features make carbon emission rights do not fit equally well into the existing framework of the auction mechanisms for ordinary multiple items. However, the existing literature, on carbon emission rights auctions, focuses on the emission reduction effectiveness and policy performance of EU ETS, leaving open associated auction mechanism design and equilibrium discussion. Thus, the auction mechanism research on carbon emission rights seriously lags behind the practice. To fill in the gap in terms of theoretical research, this paper develops a new auction mechanism for carbon emission rights by making use of the Pareto optimal concept. The result shows there exists a Pareto optimal auction equilibrium and under the condition without ties, the equilibrium is unique and efficient. The efficiency of auction mechanism is of great significance to meet the carbon emission reduction cap at the lower economic costs. In addition, the proposed mechanism consists of four steps, so it is simple to understand and easy to use, which makes it possible for future commercial applications.

Typically, to future commercial applications, the paper considers the case where the price adjustment is discrete; to theoretical completeness, the continuous situation is also analyzed. Within the proposed auction mechanism, it is found that there are a Pareto optimal equilibrium and a Pareto efficient assignment for both the discrete and continuous markets. With no-tie restriction, the Pareto optimal equilibrium and then the Pareto efficient allocation are unique in the discrete market.

Several interesting problems remain for future research in this paper. The first one is how to generalize the proposed auction to the general situation when the bidders' and the auctioneer's payoffs are nonlinear in bid prices. Another one concerns the tatonnement process which has been widely debated in economics. Since the existence and uniqueness of equilibrium for a tatonnement process are dependent on the special supply and demand functions, what the decision functions are if the bidders are heterogenous or having a more general utility function? The third one is how environmental benefit is endogenously determined by the whole economy and entered into the product functions of the bidders. The last one generalizes our mechanism to the case dynamic situation similar to Huang et al. [34].

\section{Conflict of Interests}

The authors declare that there is no conflict of interests regarding the publication of this paper.

\section{Acknowledgments}

This work was partially supported by GRF: CityU 112809 of Hong Kong SAR Government, National Natural Science Foundation of China (no. 71001097), National Social Science Foundation of China (no. 12CJY078), Program for Academic Innovative Research Team in Beijing (no. IDHT20130522), and Beijing Young Talents Plan.

\section{References}

[1] R. H. Coase, “The problem of the social cost," Journal of Law and Economics, vol. 3, pp. 1-44, 1960.

[2] T. D. Crocker, "The structuring of atmospheric pollution control systems," in The Economics of Air Pollution, H. Wolozin, Ed., pp. 61-86, W.W. Norton, New York, NY, USA, 1966.

[3] J. H. Dales, Pollution Property and Prices, Toronto University Press, Toronto, Canada, 1968. 
[4] W. L. Baunol and W. E. Oates, The Theory of Environmental Policy: Externalities, Public Outlays and the Quality of Life, Prentice-Hall, Englewood Cliffs, NJ, USA, 1988.

[5] P. Linares and C. Romero, "A multiple criteria decision making approach for electricity planning in Spain: economic versus environmental objectives," Journal of the Operational Research Society, vol. 51, no. 6, pp. 736-743, 2000.

[6] E. Georgopoulou, Y. Sarafidis, S. Mirasgedis, S. Zaimi, and D. P. Lalas, "A multiple criteria decision-aid approach in defining national priorities for greenhouse gases emissions reduction in the energy sector," European Journal of Operational Research, vol. 146, no. 1, pp. 199-215, 2003.

[7] W. D. Nordhaus, "Geography and macroeconomics: new data and new findings," Proceedings of the National Academy of Sciences of the United States of America, vol. 103, no. 10, pp. 3510$3517,2006$.

[8] F. Jotzo and J. C. V. Pezzey, "Optimal intensity targets for greenhouse gas emissions trading under uncertainty," Environmental and Resource Economics, vol. 38, no. 2, pp. 259-284, 2007.

[9] R. S. Tol, "The economic effects of climate change," Journal of Economic Perspectives, vol. 23, no. 2, pp. 29-51, 2009.

[10] Y. Zhang, "Supply-side structural effect on carbon emissions in China," Energy Economics, vol. 32, no. 1, pp. 186-193, 2010.

[11] J.-M. Burniaux and J. Oliveira Martins, "Carbon leakages: a general equilibrium view," Economic Theory, vol. 49, no. 2, pp. 473-495, 2012.

[12] K. Capoor and P. Ambrosi, State and Trends of the Carbon Market 2009, The World Bank Report, Washington, DC, USA, 2009.

[13] V. Krishna, Auction Theory, Academic Press, New York, NY, USA, 2002.

[14] P. Milgrom, "Putting auction theory to work: the simultaneous ascending auction," Journal of Political Economy, vol. 108, no. 2, pp. 245-272, 2000.

[15] L. M. Ausubel, "An efficient dynamic auction for heterogeneous commodities," The American Economic Review, vol. 96, no. 3, pp. 602-629, 2006.

[16] D. Mishra and R. Garg, "Descending price multi-item auctions," Journal of Mathematical Economics, vol. 42, no. 2, pp. 161-179, 2006.

[17] D. Mishra and D. Veeramani, "Vickrey-Dutch procurement auction for multiple items," European Journal of Operational Research, vol. 180, no. 2, pp. 617-629, 2007.

[18] A. Avenali, "Exploring the VCG mechanism in combinatorial auctions: the threshold revenue and the threshold-price rule," European Journal of Operational Research, vol. 199, no. 1, pp. 262-275, 2009.

[19] J. H. Choi, Y. S. Chang, and I. Han, "The empirical analysis of the N-bilateral optimized combinatorial auction model," Omega, vol. 37, no. 2, pp. 482-493, 2009.

[20] N. Sun and Z. Yang, "A double-track adjustment process for discrete markets with substitutes and complements," Econometrica, vol. 77, no. 3, pp. 933-952, 2009.

[21] A. Goel, M. Mahdian, H. Nazerzadeh, and A. Saberi, "Advertisement allocation for generalized second-pricing schemes," Operations Research Letters, vol. 38, no. 6, pp. 571-576, 2010.

[22] D.-H. Shih, D. C. Yen, C.-H. Cheng, and M.-H. Shih, "A secure multi-item e-auction mechanism with bid privacy," Computers and Security, vol. 30, no. 4, pp. 273-287, 2011.

[23] Q. Liu, "Equilibrium of a sequence of auctions when bidders demand multiple items," Economics Letters, vol. 112, no. 2, pp. 192-194, 2011.
[24] E. Benz and S. Truck, " $\mathrm{CO}_{2}$ emission allowances trading in Europe-specifying a new class of assets," Problems and Perspectives in Management, vol. 4, no. 3, pp. 30-40, 2006.

[25] E. Benz, A. Löschel, and B. Sturm, "Auctioning of $\mathrm{CO}_{2}$ emission allowances in phase 3 of the EU emissions trading scheme," Climate Policy, vol. 10, no. 6, pp. 705-718, 2010.

[26] P. Cramton and S. Kerr, "Tradeable carbon permit auctions how and why to auction not grandfather," Energy Policy, vol. 30, no. 4, pp. 333-345, 2002.

[27] M. Grubb, C. Azar, and U. M. Persson, "Allowance allocation in the European emissions trading system: a commentary," Climate Policy, vol. 5, no. 1, pp. 127-136, 2005.

[28] S. Clò, "Grandfathering, auctioning and carbon leakage: assessing the inconsistencies of the new ETS directive," Energy Policy, vol. 38, no. 5, pp. 2420-2430, 2010.

[29] J. Kruger and W. A. Pizer, "The EU emissions trading directive opportunities and potential pitfalls, resources for the future," Working Paper 04-24, 2004.

[30] D. Burtraw, J. Goeree, C. A. Holt, E. Myers, K. Palmer, and W. Shobe, "Collusion in auctions for emission permits: an experimental analysis," Journal of Policy Analysis and Management, vol. 28, no. 4, pp. 672-691, 2009.

[31] C. Holt, W. Shobe, D. Burtraw et al., "Auction design for selling $\mathrm{CO}_{2}$ emission allowances under the regional greenhouse gas initiative," Final Report, New York State Energy Research and Development Authority, Albany, NY, USA, 2007.

[32] F. Gul and E. Stacchetti, "Walrasian equilibrium with gross substitutes," Journal of Economic Theory, vol. 87, no. 1, pp. 95124, 1999.

[33] A. Mas-Colell, D. W. Michael, and R. G. Jerry, Microeconomic Theory, Oxford University Press, Oxford, UK, 1995.

[34] C. Huang, C. Peng, X. Chen, and F. Wen, "Dynamics analysis of a class of delayed economic model," Abstract and Applied Analysis, vol. 2013, Article ID 962738, 12 pages, 2013. 


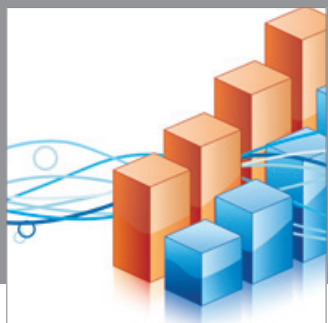

Advances in

Operations Research

mansans

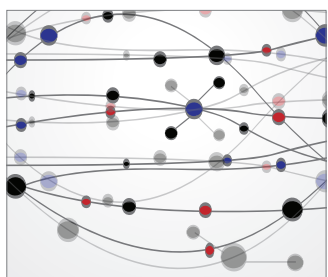

The Scientific World Journal
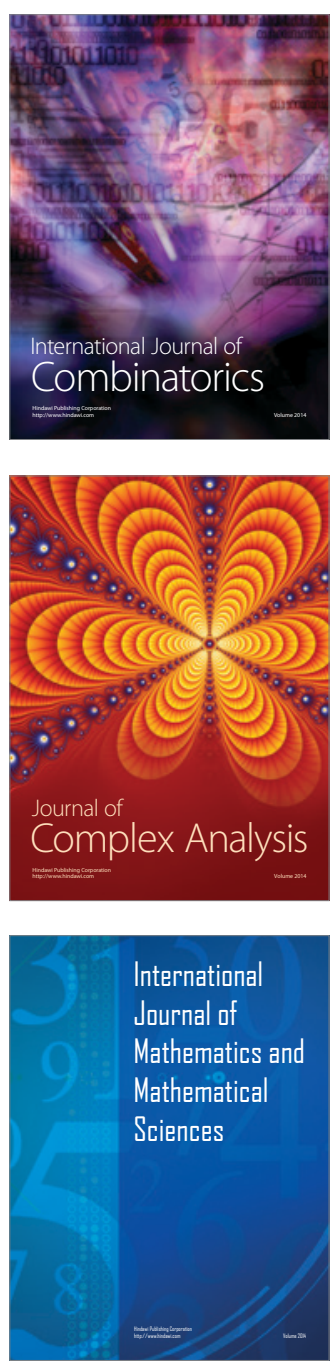
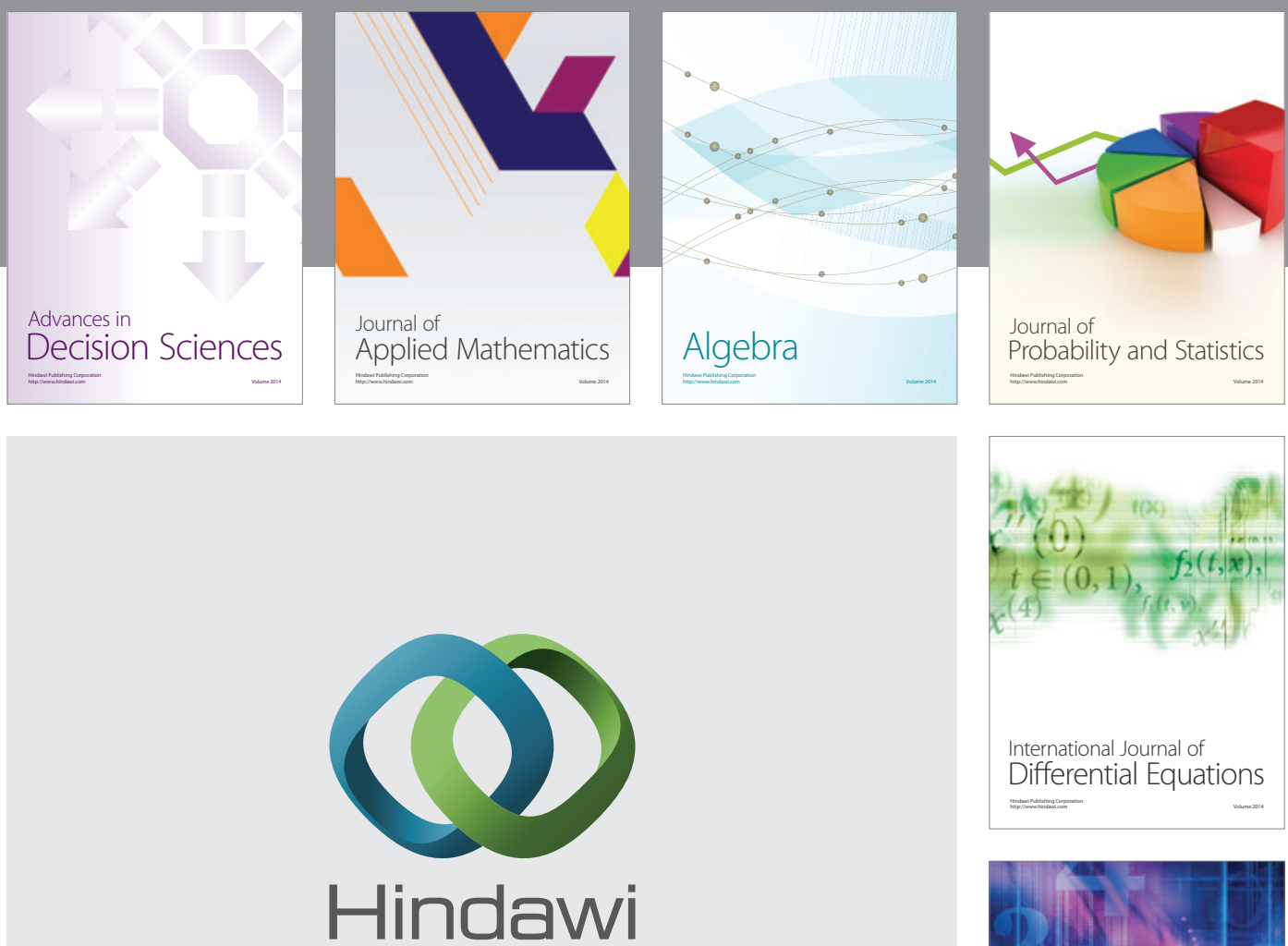

Submit your manuscripts at http://www.hindawi.com
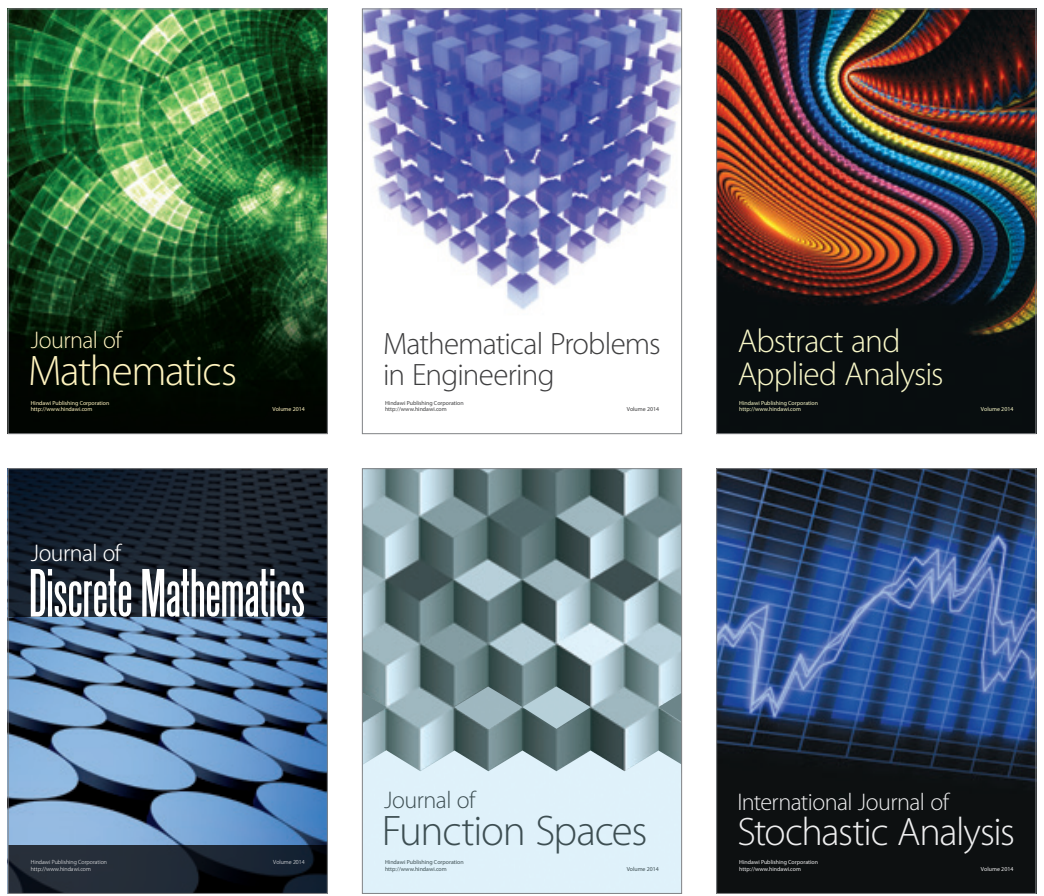

Journal of

Function Spaces

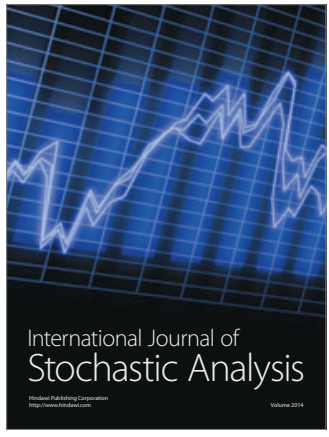

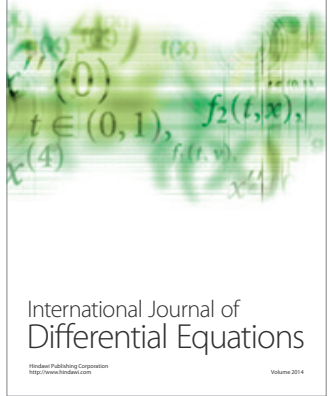
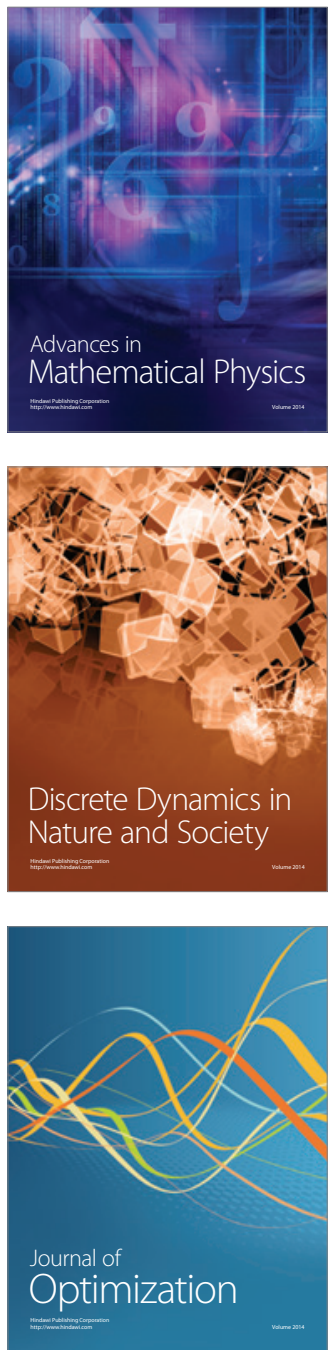